Formation et évolution des sols sur matériaux calcaires dans le piémont appalachien, Québec

Soil Formation and Evolution on Calcareous Parent Materials in the Appalachian Piedmont, Québec Bodenbildung und -entwicklung auf kalkhaltigem Material im Appalachen Piémont, Québec

\author{
Christian R. De Kimpe, Marc R. Laverdière, Gérard Laflamme et Michel \\ Rompré
}

Volume 39, numéro 3, 1985

URI : https://id.erudit.org/iderudit/032610ar

DOI : https://doi.org/10.7202/032610ar

\section{Aller au sommaire du numéro}

\section{Éditeur(s)}

Les Presses de l'Université de Montréal

\section{ISSN}

0705-7199 (imprimé)

1492-143X (numérique)

\section{Découvrir la revue}

Citer cet article

De Kimpe, C. R., Laverdière, M. R., Laflamme, G. \& Rompré, M. (1985). Formation et évolution des sols sur matériaux calcaires dans le piémont appalachien, Québec. Géographie physique et Quaternaire, 39(3), 299-306. https://doi.org/10.7202/032610ar

\section{Résumé de l'article}

Les sols développés sur matériaux calcaires et qualifiés de résiduels à semi-résiduels par les pédologues occupent plus de 15000 ha au Québec. Quatre profils ont été échantillonnés près de Plessisville, dans un milieu de dépôts glaciaires, pour étudier la formation et l'évolution de tels sols. Les dépôts meubles ne dépassent pas 1 mètre d'épaisseur. Les schistes sont absents du solum des profils modérément bien drainés, tandis que leur contenu augmente depuis la surface vers la profondeur dans le profil mal drainé. Des mesures de désagrégation des schistes dans l'eau et HCI dilué ont montré que la décomposition des schistes est favorisée par la dissolution du carbonate. L'instabilité des schistes dans l'eau semble pouvoir expliquer le caractère résiduel des dépôts meubles. Pour connaître leur origine, glaciaire ou résiduelle, il paraît nécessaire d'étudier la relation roche arène dans un dépôt profond. Dans les profils modérément bien drainés, il y a formation de complexes organométalliques de $\mathrm{Fe}$ et $\mathrm{Al}$, mais peu de transformation des minéraux. Un podzol humo-ferrique orthique et un brunisol mélanique orthique ont été identifiés. Le sol imparfaitement drainé montre peu de différenciation à l'intérieur du profil et il a été classé comme un brunisol mélanique gleyifié. Le carbonate éliminé a été transporté latéralement et s'est accumulé sous forme de marne dans la dépression voisine. Un humisol terrique s'est développé à cet endroit. 


\title{
FORMATION ET ÉVOLUTION DES SOLS SUR MATÉRIAUX CALCAIRES DANS LE PIÉMONT APPALACHIEN, QUÉBEC*
}

\begin{abstract}
Christian R. DE KIMPE, Marc R. LAVERDIÈRE, Gérard LAFLAMME et Michel ROMPRÉ; premier auteur : Agriculture Canada, Station de recherches, 2560, boulevard Hochelaga, Sainte-Foy, Québec G1V 2J3; deuxième auteur: Département des sols, Université Laval, Sainte-Foy, Québec G1K 7P4; troisième et quatrième auteurs: Service de recherches en sols, Ministère de l'Agriculture, des Pêcheries et de l'Alimentation du Québec, 2700, rue Einstein, Sainte-Foy, Québec G1P 3 W8.
\end{abstract}

RÉSUMÉ Les sols développés sur matériaux calcaires et qualifiés de résiduels à semirésiduels par les pédologues occupent plus de 15000 ha au Québec. Quatre profils ont été échantillonnés près de Plessisville, dans un milieu de dépôts glaciaires, pour étudier la formation et l'évolution de tels sols. Les dépôts meubles ne dépassent pas 1 mètre d'épaisseur. Les schistes sont absents du solum des profils modérément bien drainés, tandis que leur contenu augmente depuis la surface vers la profondeur dans le profil mal drainé. Des mesures de désagrégation des schistes dans l'eau et $\mathrm{HCl}$ dilué ont montré que la décomposition des schistes est favorisée par la dissolution du carbonate. L'instabilité des schistes dans l'eau semble pouvoir expliquer le caractère résiduel des dépôts meubles. Pour connaître leur origine, glaciaire ou résiduelle, il paraît nécessaire d'étudier la relation roche arène dans un dépôt profond. Dans les profils modérément bien drainés, il y a formation de complexes organométalliques de $\mathrm{Fe}$ et $\mathrm{Al}$, mais peu de transformation des minéraux. Un podzol humo-ferrique orthique et un brunisol mélanique orthique ont été identifiés. Le sol imparfaitement drainé montre peu de différenciation à l'intérieur du profil et il a été classé comme un brunisol mélanique gleyifié. Le carbonate éliminé a été transporté latéralement et s'est accumulé sous forme de marne dans la dépression voisine. Un humisol terrique s'est développé à cet endroit.
ABSTRACT Soil formation and evolution on calcareous parent materials in the Appalachian piedmont, Québec. Soils developed on calcareous parent materials and identified as "residual or semi-residual" by soil surveyors, cover more than 15000 ha in Québec. Four profiles were sampled near Plessisville, in a once glaciated area, in order to study soil formation. Loose parent material over the rock did not exceed one meter. Shale fragments were absent in the moderately well drained profiles whereas the content increased with depth in the poorly drained one. Disaggregation tests carried out in water and dilute $\mathrm{HCl}$ indicated that the shale decomposed more easily when carbonate was dissolved. Shale disaggregation could account for the residual character of the parent material, as proposed by several authors. However, to assign more definitely a glacial or residual origin to the parent material in this area, it would be necessary to investigate the rock-arenite transition in a deeper deposit. In the moderately well drained profiles, mineral alteration was moderate and organometallic complexes were translocated to the $\mathrm{B}$ horizons. One profile was classified as an orthic humo-ferric podzol and the second one as an orthic melanic brunisol. There was only very slight profile development in the poorly drained soil that was classified as a gleyed melanic brunisol. Carbonate dissolved in the profiles moved to an adjacent depression, where it accumulated as a marl deposit; here a terric humisol developed.
ZUSAMMENFASSUNG Bodenbildung und -entwicklung auf kalkhaltigem Material im Appalachen Piémont, Québec. Die Böden, die sich auf kalkhaltigem Material entwickelt haben, und die von den Bodenforschern als Rückstandsböden bis Halbrückstandsböden bezeichnet werden, nehmen mehr als 15000 ha in Québec ein. Bei Plessisville sind in einem Milieu eiszeitlicher Ablagerungen vier ProfilProben entnommen worden, um die Bildung und Entwicklung solcher Böden zu studieren. Die lockeren Ablagerungen sind nicht mehr als ein Meter tief. Im Boden der ziemlich gut entwässerten Profile befinden sich keine Schiefer, wohingegen der Schiefer-Anteil in den schlecht entwässerten Profilen von der Oberfläche bis in die Tiefe zunimmt. In Wasser und verdünntem $\mathrm{HCl}$ durchgeführte ZerfallTests der Schiefer haben gezeigt, da $\beta$ der Zerfall der Schiefer durch die Auflösung des Karbonats begünstigt wird. Die Labilität der Schiefer im Wasser scheint den Rückstandscharakter der lockeren Ablagerungen zu erklären. Um ihre eiszeitliche oder residuale Herkunft zu kennen, scheint es notwendig, die Beziehung Felsen-Grus in einer tiefgelegenen Ablagerung zu studieren. In den mäBig gut entwässerten Profilen findet man Organimetall-Komplexe von $\mathrm{Fe}$ und $\mathrm{Al}$, aber wenig Verwandlung der Minerale. Ein «orthic humo-ferric" Podsol und ein "orthic melanic brunisol" wurden identifiziert. Der schlecht entwässerte Boden weist wenig Differenzierung im Innern des Profils auf, und er wurde als ein "gleyed melanic brunisol" eingeordnet. Das eliminierte Karbonat ist seitwärts transportiert worden und hat sich in Form von Mergel in der Nachbarsenke angesammelt. An dieser Stelle hat sich ein erdiger Humusboden entwickelt. 


\section{INTRODUCTION}

La nature de la roche mère est un facteur important dans la formation des sols et l'intensité de leur développement. Ainsi, les mécanismes de formation sont ralentis et les profils de sol peu développés tant que le matériau parental meuble contient des quantités élevées de carbonates (ALIAS et HERNANDEZ, 1981). Les sols les plus fréquemment observés sur matériaux calcaires, en milieu bien à modérément bien drainés, sont les luvisols, qui résultent de la migration d'argile vers les horizons B (COMMISSION CANADIENNE DE PÉDOLOGIE, 1978). Ces sols prédominent sur plus de $10 \%$ du territoire canadien (BENTLEY, 1979). On les retrouve dans toutes les provinces et ils présentent des degrés divers de développement et d'altération minéralogique (McKEAGUE et al., 1972; ACTON et al., 1978; ST-ARNAUD et SUDOM, 1981).

La genèse des sols sur matériaux calcaires ou associés à ces derniers a été peu étudiée au Québec. En raison d'un climat subhumide de type continental tempéré à hiver froid et été chaud (ATLAS NATIONAL DU CANADA, 1972) environ la moitié des précipitations sont sous forme de pluie et le lessivage intense des cations alcalins et alcalino-terreux hors du profil est phénomène courant. Lorsque la teneur en carbonates est suffisamment réduite, les processus de formation agissent et le développement du profil progresse rapidement.

Ainsi LAFLAMME et al. (1973) ont décrit un podzol humoferrique, luvisolique et lithique, développé dans les schistes à calcaire rubanné de la formation Trinité, dans le comté de Rimouski. Ces auteurs ont montré que le front de la podzolisation suivait de fort près l'élimination des carbonates, mais ils ont aussi souligné l'effet de l'entraînement de l'argile sur le développement du profil. Dans les situations où la pédogenèse se poursuit longtemps et de façon assez intense, on peut même observer des profils bisequa (McKEAGUE et al., 1967).

Le présent travail comportait deux objectifs. En premier lieu, il visait à obtenir une meilleure connaissance des sols du Québec développés sur des matériaux calcaires schisteux ou ardoisiers dans lesquels le fait calcaire est plus uniformément distribué que dans le cas des schistes à calcaire rubané. De plus, beaucoup de sols développés sur ces matériaux furent qualifiés par les pédologues de résiduels, quasirésiduels ou semi-résiduels (BARIL et ROCHEFORT, 1957; CHOINIĖRE, 1960; RAYMOND et al., 1976; ROMPRÉ et al., 1984) et il est apparu utile de chercher quelques critères qui pourraient préciser l'origine du matériau meuble qui a donné naissance à ces sols.

\section{MATÉRIAUX ET MÉTHODES}

\section{GÉOLOGIE}

La formation de Bullstrode, composée d'ardoises calcareuses et graphitiques gris noir et d'interlits de calcaire graphitique gris noirâtre a été décrite dans la région de Drummondville (GLOBENSKY, 1978). Cette formation se prolonge sous forme d'une large bande orientée SO-NE jusque dans les comtés d'Arthabaska et de Mégantic et elle est la continuation du calcaire de Melbourne décrit dans la région de Warwick (COOKE, 1952). Elle date de L'Ordovicien.

Du point de vue pétrographique, les calcaires se composent de calcaire argileux, gris en surface fraîche, gris terne à gris brunâtre en surface altérée (GLOBENSKY, 1978). Ils se présentent en lits de 5 à $60 \mathrm{~cm}$ d'épaisseur et contiennent, en plus des carbonates, du quartz, des feldspaths et des phyllosilicates dont la nature n'est pas précisée. Les ardoises ont une même couleur gris noir à l'état frais et gris brunâtre en surface altérée. Leur épaisseur varie de 1 à $10 \mathrm{~cm}$.

\section{CLIMAT}

À Victoriaville, à $24 \mathrm{~km}$ au sud-ouest de Plessisville, située à $150 \mathrm{~m}$ d'altitude dans le piémont des Appalaches, la température moyenne annuelle est de $4,9^{\circ} \mathrm{C}$, alors que la température moyenne en juillet est de $19,2^{\circ} \mathrm{C}$. Les précipitations moyennes annuelles sont de $109 \mathrm{~cm}$ dont $49 \mathrm{~cm}$ entre mai et septembre (ENVIRONNEMENT CANADA, 1970).

\section{ÉCHANTILLONNAGE}

L'échantillonnage a été effectué au sud de Plessisville, dans le comté de Mégantic, à $46^{\circ} 11^{\prime} \mathrm{N}$ et $75^{\circ} 45^{\prime} \mathrm{O}$, le long d'une pente de 3 à $5 \%$, longue de 250 m environ et exposée au nord-est. L'altitude du site était de $175 \mathrm{~m}$. Les profils ont été prélevés dans des champs cultivés sur une crête légèrement bombée (profil Melbourne), vers le milieu de la pente (profil intermédiaire Melbourne-Savoie) et sur le bord d'une dépression (sol organique), au bas de la pente. Un second profil Melbourne a été échantillonné sur une crête à $2 \mathrm{~km}$ environ au nord du site précédent et à la même altitude. Dans les profils Melbourne et Melbourne-Savoie, le sol était perméable, mais les sites étaient respectivement modérément bien et imparfaitement drainés, à cause de la proximité de la roche sous-jacente. Le sol organique était dans un site très mal drainé.

La description des profils est la suivante:

$\begin{array}{ccc}\text { Horizon } & \begin{array}{c}\text { Profondeur } \\ (\mathrm{cm})\end{array} & \text { Description }\end{array}$

\section{Site $n^{\circ} 1$ Profil Melbourne}

Ap $\quad 0-21$ Loam brun foncé (10 YR $3 / 3 \mathrm{~h}$ ), brun olive clair ( 2.5 Y $5 / 4 \mathrm{~s})$; granulaire, faible très friable; racines très abondantes, grossières à fines; limite abrupte, régulière; $\mathrm{pH}\left(\mathrm{H}_{2} \mathrm{O}\right)$ : 6,5 .

$\mathrm{Bf}(\mathrm{Bmtj}) \quad 21-43 \quad$ Loam limoneux brun jaune foncé (10 YR $4 / 4 \mathrm{~h}$ ), brun jaune clair (2.5 Y $6 / 4 \mathrm{~s})$; polyédrique, subangulaire, fine, faible: friable; pellicules argileuses, peu communes, très minces; racines peu abondantes, fines à très fines; limite graduelle, régulière; $\mathrm{pH}: 6,2$.

$\mathrm{BC}_{1} \quad 43-62 \quad$ Loam limoneux brun olive $(2.5 \mathrm{Y} 4 / 4 \mathrm{~h})$, brun jaune clair (2.5 Y 6/4 s); pseudolamellaire; friable; racines très peu abondantes, très fines; limite diffuse, régulière; $\mathrm{pH}: 6,2$. 


\begin{abstract}
$\mathrm{BC}_{2} \quad 62-81 \quad$ Loam limoneux brun olive (2.5 Y $\left.4 / 4 \mathrm{~h}\right)$, brun olive clair ( 2.5 Y $5 / 6 \mathrm{~s}$ ); pseudolamellaire; ferme à friable; racines très peu abondantes, très fines; limite diffuse, irrégulière; $\mathrm{pH}: 6,8$.

Ck $81-100$ Loam limoneux brun olive (2.5 Y $4 / 4 \mathrm{~h})$, brun olive clair ( 2.5 Y $5 / 4 \mathrm{~s}$ ); pseudolamellaire; ferme; pas de racines; effervescence forte avec $\mathrm{HCl} 10 \%$; $\mathrm{pH}$ : 8,0 .

Rk $\quad 100+$

Roche décomposée gris très foncé (2.5 Y $3 / 0 \mathrm{~h}$ ), effervescence forte avec $\mathrm{HCl} 10 \% ; \mathrm{pH}: 8,3$.
\end{abstract}

Site $n^{\circ} 2$ Profil Melbourne

Ap $\quad 0-20 \quad$ Loam limoneux brun foncé (10 YR 3/ $3 \mathrm{~h})$, brun olive clair ( 2.5 Y $5 / 4 \mathrm{~s})$; granulaire; très friable; racines très abondantes, grossières à très fines; limite abrupte, régulière; $\mathrm{pH}: 6,4$.

$\mathrm{Bm}$ (Bfjtj) $\quad 20-30 \quad$ Loam limono-argileux brun jaune foncé (10 YR 4/4 h), brun olive clair (2.5 Y 5/ $6 \mathrm{~s})$; polyédrique subangulaire fine, très faible; friable; pellicules argileuses, peu communes, très minces; racines abondantes, fines à très fines; limite nette, régulière; $\mathrm{pH}: 6,6$.

BC $\quad 30-58$ Loam limono-argileux olive (5 Y $5 / 3 \mathrm{~h}$ ), brun jaune clair ( 2.5 Y $6 / 4 \mathrm{~s}$ ); polyédrique subangulaire; racines peu abondantes, très fines; limite graduelle, régulière; $\mathrm{pH}$ : 7,4 .

$\mathrm{Ck}_{1} \quad 58-80 \quad$ Loam limoneux olive $(5 \mathrm{Y} 5 / 3 \mathrm{~h})$, gris brun clair (2.5 Y $6 / 2 \mathrm{~s})$; pseudolamellaire; racines très peu abondantes, très fines; effervescence modérée; limite diffuse, irrégulière; $\mathrm{pH}: 8,1$.

$\mathrm{Ck}_{2} \quad 80-100$ Loam limoneux gris olive $(5 \mathrm{Y} 5 / 2 \mathrm{~h})$, brun jaune clair ( 2.5 Y 6/4 s); pseudolamellaire; pas de racines; effervescence modéree avec $\mathrm{HCl} 10 \%$; limite nette, irrégulière; $\mathrm{pH}: 8,2$.

Rk $110+\quad$ Roche décomposée olive (5 Y $4 / 3 \mathrm{~h}$ ); effervescence forte avec $\mathrm{HCl} 10 \%$; $\mathrm{pH}$ : 8,3 .

\section{Site $n^{\circ} 1$ Profil intermédiaire Melbourne-Savoie}

Ap $\quad 0-14 \quad$ Loam limoneux brun gris très foncé (10 YR $3 / 2 \mathrm{~h}$ ), brun gris foncé (2.5 Y 4.5 / $2 \mathrm{~s})$; granulaire; très friable; racines très abondantes, grossières à fines; limite abrupte, régulière; $\mathrm{pH}: 7,1$.

Bmgj $\quad 14-24$ Loam limoneux olive ( 5 Y $5 / 4 \mathrm{~h}$ ), gris clair (2.5 Y $7 / 2 \mathrm{~s})$; pseudopolyédrique subangulaire; friable; marbrures nombreuses, moyennes, distinctes à faibles: racines très peu abondantes, fines à très fines: $10 \%$ de gravier anguleux; limite nette, ondulée; $\mathrm{pH}: 7,2$.

Cgj, $\quad 24-34$ Loam limoneux olive (5 Y $4 / 3 \mathrm{~h}$ ), gris brun clair (2.5 Y $6 / 2 \mathrm{~s}$ ); pseudolamellaire; ferme; marbrures rares, petites, faibles; racines très peu abondantes, très fines;
$50 \%$ de gravier anguleux; limite graduelle régulière; $\mathrm{pH}: 7,4$.

\begin{tabular}{|c|c|c|}
\hline $\mathrm{Cgj}_{2}$ & $34-50$ & $\begin{array}{l}\text { Loam limoneux brun gris foncé }(2.5 \mathrm{Y} \\
4 / 2 \mathrm{~h}) \text {, gris brun clair }(2.5 \mathrm{Y} 6 / 2 \mathrm{~s}) \text {; } \\
\text { pseudolamellaire; ferme; marbrures } \\
\text { rares, petites, faibles; racines très peu } \\
\text { abondantes, très fines; } 70 \% \text { de gravier } \\
\text { anguleux; limite graduelle, régulière; } \mathrm{pH} \text { : } \\
7,6 \text {. }\end{array}$ \\
\hline Ckgj & $50-56$ & $\begin{array}{l}\text { Loam limoneux brun jaune foncé ( } 10 \mathrm{YR} \\
4 / 4 \mathrm{~h} \text { ), brun jaune ( } 10 \mathrm{YR} 5 / 6 \mathrm{~s} \text { ), pseu- } \\
\text { dolamellaire; marbrures rares, petites, } \\
\text { faibles; pas de racines; } 80 \% \text { de gravier } \\
\text { anguleux; effervescence forte avec } \mathrm{HCl} \\
10 \% \text {; } \mathrm{pH}: 8,0 \text {. }\end{array}$ \\
\hline Rk & $56+$ & $\begin{array}{l}\text { Roche altérée brun gris foncé }(2.5 \mathrm{Y} 4 \\
2 \mathrm{~h}) ; \text { effervescence très forte avec } \mathrm{HCl} \\
10 \% ; \mathrm{pH}: 8,3 \text {. }\end{array}$ \\
\hline
\end{tabular}

Site $n^{\circ} 1$ Sol organique

Op $\quad 0-24$

Couche organique bien décomposée rouge très foncé (2.5 YR 2.5/2 h), brun rouge foncé ( 5 YR $2.5 / 2 \mathrm{~s}$ ); granulaire, très faible; racines abondantes; limite nette, régulière; $\mathrm{pH}: 6,6$.

Oh, 24-32 Couche organique bien décomposée noire (5 YR 2.5/1 h), brun rouge foncé (5 YR 2.5/2 s); granulaire, très faible; racines abondantes; limite diffuse, régulière; $\mathrm{pH}: 6,6$.

$\mathrm{Oh}_{2} \quad 32-42 \quad$ Couche organique bien décomposée noire (5 YR 2.5/1 h), brun foncé (7.5 YR $3 / 2 \mathrm{~s})$; granulaire, faible; racines abondantes; limite abrupte, régulière; $\mathrm{pH}: 7.0$.

Ck $\quad 42-52 \quad$ Loam limoneux gris clair $(2.5 \mathrm{Y} 7 / 2 \mathrm{~h})$, blanc $(2.5$ Y $8 / 1 \mathrm{~s})$; granulaire; ferme; pas de racines; effervescence forte avec $\mathrm{HCl} 10 \%$; limite nette, régulière; $\mathrm{pH}: 7,8$.

IICcag $52+\quad$ Sable loameux gris très foncé $(2.5$ Y 3 / $0 \mathrm{~h})$, gris (2.5 Y 5/0 s); massif; pas de racine; effervescence modérée avec $\mathrm{HCl}$ $10 \% ; \mathrm{pH}: 6,9$.

\section{ANALYSES}

Les méthodes d'analyse utilisées ont pour la plupart été tirées du manuel de méthodes d'échantillonnage et d'analyse des sols (McKEAGUE, 1978). Elles sont indiquées avec leur numéro de référence; texture: méthode de la pipette (2.11) - pH: dans $\mathrm{H}_{2} \mathrm{O}$ (3.13) et $\mathrm{CaCl}_{2} 0.01 \mathrm{M}$ (3.11) - carbone organique: oxydation par voie humide (3.613) - carbonates: dissolution par $\mathrm{HCl}$ et titration en retour par $\mathrm{NaOH}$ - sesquioxydes de $\mathrm{Fe}$ et $\mathrm{Al}$ : extraction par le dithionite-citratebicarbonate (3.51) et le pyrophosphate (3.53) - capacité d'échange cationique: $\mathrm{NH}_{4} \mathrm{OAc}(3.34)$ - bases échangeables: extraction avec $\mathrm{NaCl}$ (3.31) - analyse minéralogique: diffraction des rayons $X$ en utilisant des sous-échantillons d'argile saturée avec Mg ou K, préparés sous forme de lames orientées (5.22)

La stabilité du gravier dans l'eau a été déterminée selon la méthode décrite par RIVARD et DE KIMPE (1980). Le même test a été effectué en remplaçant l'eau par $\mathrm{HCl} 0,1 \mathrm{~N}$. 
Dans ce cas, après chaque cycle d'agitation, l'échantillon a été rincé à l'eau avant d'être séché et tamisé.

\section{RÉSULTATS ET DISCUSSION}

\section{MATÉRIAU PARENTAL ET STABILITÉ DES SCHISTES}

Les séries de sol Melbourne et Savoie ont été décrites par ROMPRÉ et al. (1984) dans le comté d'Arthabaska. Elles ont été cartographiées aussi dans le comté de Mégantic (rapport en cours de rédaction). Selon ces auteurs, il s'agit de sols développés sur des dépôts de till incorporant des éléments de la formation Melbourne sous-jacente. La nature des dépôts serait donc morainique. Les formes de terrain, pentes longues, croupes basses et sommets arrondis, montrent de façon nette le passage des glaciers dans la région. De plus, la présence de sols dérivés de till (séries Francœur, Raimbault, Bedford et Kingsey) que l'on trouve intercalés dans l'aire de distribution des sols Melbourne et Savoie dans le comté d'Arthabaska (ROMPRÉ et al., 1984), corrobore cet énoncé. Comme les schistes calcaires sont moins résistants à l'érosion glaciaire que les roches gréseuses ou quartzeuses que l'on trouve ailleurs dans les Appalaches, le relief plus adouci qui en est résulté peut expliquer que la couverture de till est généralement mince.

Toutefois, il ne faudrait pas ignorer la possibilité d'une origine résiduelle pour les matériaux meubles qui ont donné naissance aux sols étudiés ici. Cette hypothèse a aussi été mentionnée par les auteurs du rapport (ROMPRÉ et al., 1984). L'absence de pierres ou de graviers allochtones serait un indice. Le caractère résiduel ou semi-résiduel des sols peut être déterminé, entre autres, par le degré de décomposition de la roche mère et par la facilité avec laquelle la roche peut être arénisée. Dans son ensemble, la formation de Bullstrode présente un pendage qui varie de l'horizontale à la verticale (GLOBENSKY, 1978). Dans la région de Plessisville, l'orientation presque verticale, observée en plusieurs endroits, favorise certainement la décomposition de la roche et le lessivage par l'eau. L'effet du pendage des schistes sur la formation de sols résiduels a déjà été abordé pour les schistes Utica dans les comtés de Lotbinière (BARIL et ROCHEFORT, 1957) et de Portneuf (RAYMOND et al., 1976). En effet, lorsque les schistes se trouvent en position quasi-horizontale, les sols Tilly, Joly et Platon, qui s'y sont développés, reposent en contact abrupt sur un "dallage" comportant un réseau faiblement développé de diaclases étroites. Par contre, quand le pendage est plus marqué, les schistes sont plus fracturés et le contact entre le sol et la roche se fait par l'intermédiaire d'une zone de roche pourrie.

La transformation des schistes Melbourne en matériau meuble fin a été mesurée par des essais de stabilité. Les tests ont été réalisés sur des échantillons prélevés dans divers horizons des profils Melbourne et Melbourne-Savoie, et menés dans l'eau et $\mathrm{HCl}$ dilué (fig. 1). Cinq cycles agitation dans l'eau (ou $\mathrm{HCl}$ ) - séchage - tamisage ont été appliqués successivement. L'examen de la figure 1 appelle quelques commentaires:

a) la stabilité des schistes croît avec la profondeur de prélèvement; b) un écart important entre les lignes représentant le gravier résiduel $>6 \mathrm{~mm}$ après agitation dans l'eau et $\mathrm{HCl} 0,1 \mathrm{~N}$ apparaît pour les horizons profonds, encore riches en $\mathrm{CaCO}_{3}$. La différence est d'environ $20 \%$ pour les échantillons provenant des horizons $\mathrm{Ck}$ et Rk du profil Melbourne 1 et Rk du profil Melbourne-Savoie;

c) les schistes provenant des profils modérément bien drainés (Melbourne) sont moins stables que ceux provenant du profil imparfaitement drainé (Melbourne-Savoie).

II apparaît donc qu'un drainage adéquat du milieu est suffisant pour entraîner la décomposition des fragments schisteux dans les horizons supérieurs des profils et ameublir le matériau en profondeur. Ceci explique aussi leur présence jusque près de la surface dans les profils moins bien drainés (profil Melbourne-Savoie) et renforce donc l'hypothèse d'une origine à caractère résiduel pour le matériau parental des profils étudiés.

Les sols issus de matériaux calcaires et qualifiés de quasirésiduels ou résiduels occupent plus de $150 \mathrm{~km}^{2}$ au Québec, tant dans les basses terres que dans le piémont appalachien (séries Tilly, Joly, Platon, Henryville, Kingsey mince, Melbourne et Savoie). II s'agit dans les deux régions de zones assez vastes et il ne serait donc pas étonnant qu'on puisse trouver des profils développés dans du till libre de matériaux allochtones et dans lesquels, en milieu bien drainé, les fragments grossiers ont été complètement décomposés. La persistance de charges grossières et, surtout, de schistes non orientés dans les sols moins bien drainés pourrait suggérer la présence d'un till, mais il faut se rappeler que, puisque les sols sont minces, la non-orientation des schistes pourrait aussi résulter de la cryoturbation. Dans l'état actuel de la question, il est donc difficile de trancher le débat. II paraît indispensable de considérer la mise en place de ces dépôts meubles dans un contexte géographique régional et il serait souhaitable de découvrir quelques sites où l'épaisseur des dépôts meubles atteindrait au moins $2 \mathrm{~m}$, afin de pouvoir étudier la filiation roche mère - arène en éliminant les facteurs de cryoturbation.

\section{DÉVELOPPEMENT DES PROFILS ET CLASSIFICATION}

\section{Profils Melbourne}

Les deux profils sont caractérisés par l'importance du limon, qui dépasse généralement $60 \%$ (tabl. I). Ce pourcentage décroît vers la surface, alors qu'augmente la teneur en argile. Dans les deux profils, la teneur en argile atteint un maximum dans l'horizon $\mathrm{B}$, ce qui est attribué, du moins en partie, à une illuviation d'argile venant de l'horizon Ap. Le rapport argile fine/argile totale dans les horizons $B$ (de 0,2 à 0,3 ) est légèrement supérieur à celui trouvé dans les horizons $C(0,2$ en moyenne). Mais l'absence d'un horizon Ae ne permet pas de vérifier la migration de l'argile. Celle-ci était plus évidente au site $n^{\circ} 1$ qu'au site $n^{\circ} 2$. Les revêtements d'argile sur les surfaces des peds étaient aussi peu communs et très minces. Ceci est en accord avec des analyses de McKEAGUE et al., (1978) qui ont montré que la quantité d'argile illuviale était inférieure à $0,2 \%$ dans le tiers des horizons Bt qu'ils ont examinés. Du point de vue chimique, la teneur en carbone organique décroît rapidement avec la profondeur. Bien que 


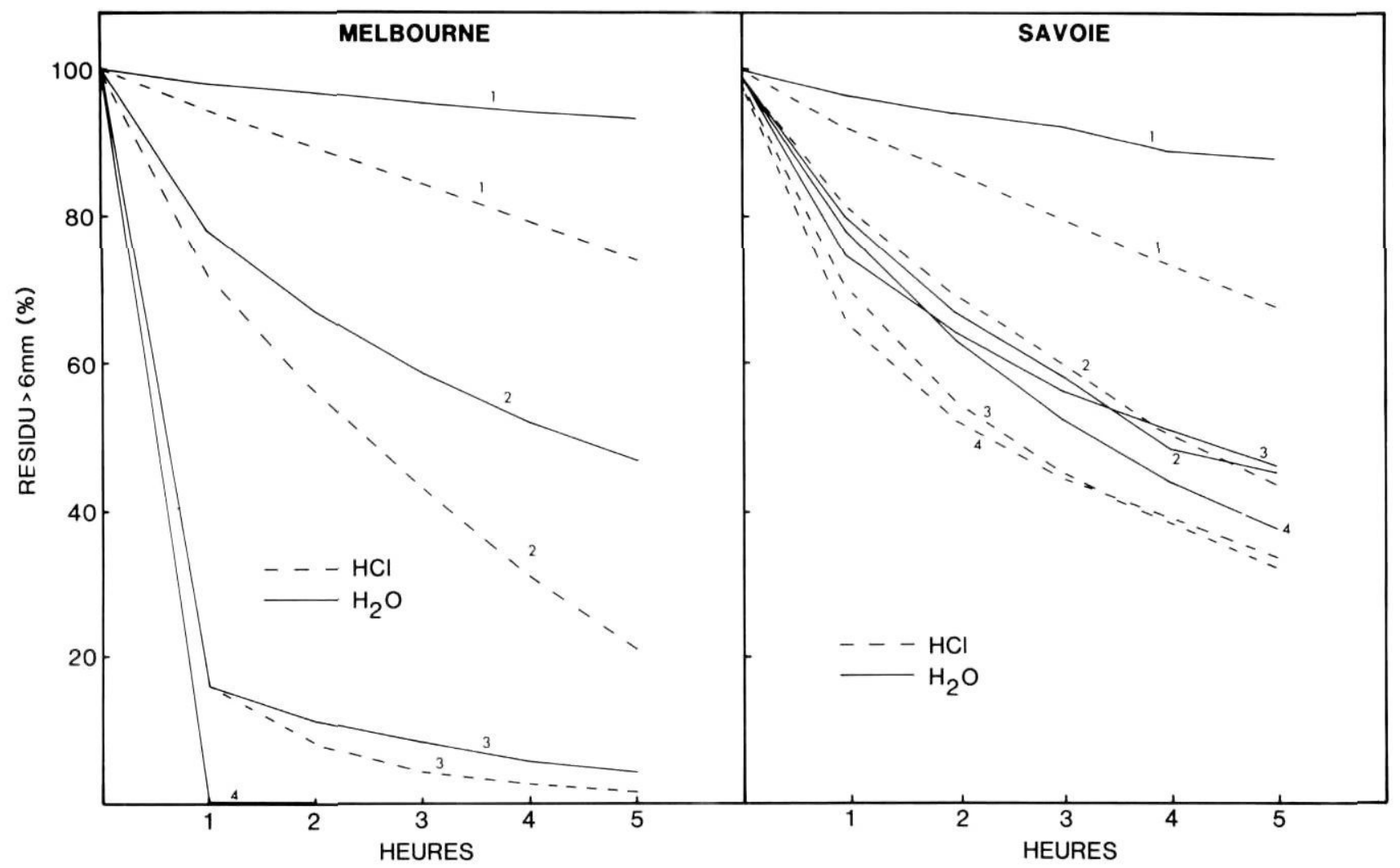

FIGURE 1. Essais de désagrégation des schistes séparés dans divers horizons des profils Melbourne 1 et Melbourne-Savoie. Melbourne: 1: Rk-2: $\mathrm{Ck}_{-}-3: \mathrm{BC}_{2}-4: \mathrm{BC}_{1}$ Savoie: $1: \mathrm{Rk}-2: \mathrm{Cg}_{3}-3: \mathrm{Cg}_{2}-4: \mathrm{Cg}_{1}$

les carbonates soient complètement éliminés des horizons supérieurs, les valeurs de $\mathrm{pH}$ dans l'eau restent supérieures à 6. Les valeurs de $\mathrm{pH}$ dans $\mathrm{CaCl}_{2} 0.01 \mathrm{M}$ pour les horizons $A$ et $B$ sont inférieures à 6 , mais la différence entre les valeurs de $\mathrm{pH}$ mesurées dans cette solution et dans l'eau sont attribuables à un effet de sel sur le déplacement d'ions acidifiants. Les ions échangeables sont représentés à plus de $90 \%$ par $\mathrm{Ca}^{2+}$ et le taux de saturation en bases est élevé, même dans les horizons supérieurs.

Les mesures de capacité d'échange cationique (CEC) ont été effectuées sur le sol entier $(<2 \mathrm{~mm}$ ) ainsi que sur l'argile $(<2 \mu \mathrm{m})$ et le limon $(50-2 \mu \mathrm{m})$. La CEC du limon représente en moyenne le tiers de celle de l'argile, ce qui est important pour la fertilité du sol si l'on considère le pourcentage de limon dans ces profils. On peut attribuer cette CEC à la nature même du matériau car, au cours de l'altération, les particules les plus fines de la fraction limon sont partiellement transformées et contribuent donc à la CEC totale du sol.

Les quantités de fer extraites par le dithionite-citrate-bicarbonate dépassent $5 \%$ dans l'horizon B supérieur. Dans I'horizon $\mathrm{BC}_{2}$ surmontant l'horizon calcaire du site $\mathrm{n}^{\circ} 1$, un pourcentage supérieur à $5 \%$ peut être attribué à une accumulation de fer due au mouvement latéral de l'eau au-dessus d'un horizon plus compact et à des changements dans les
Disaggregation tests carried out on shales from various horizons in profiles Melbourne 1 and Melbourne-Savoie. conditions d'oxydo-réduction. Ceci était moins net dans le cas du site $n^{\circ} 2$, à cause de la moins grande épaisseur de l'ensemble des horizons B et BC. Les quantités d'aluminium extrait par le dithionite-citrate-bicarbonate sont faibles. La somme des pourcentages de fer et d'aluminium extraits par le pyrophosphate dans les horizons $B$ atteint et même dépasse $1 \%$, ce qui indique la formation de complexes organométalliques.

Du point de vue minéralogique, dans la fraction argileuse, on retrouve en profondeur l'illite comme minéral principal, la chlorite et le talc comme minéraux accessoires, ainsi que de faibles quantités de quartz et de feldspaths. L'altération des minéraux dans les horizons supérieurs est faible et représentée par une diminution de la chlorite et la formation de minéraux interstratifiés illite-smectite. On a déjà observé ce phénomène dans d'autres profils sur matériaux carbonatés (ALIAS et HERNANDEZ, 1981).

En ce qui concerne la classification, le profil prélevé au site $\mathrm{n}^{\circ 1}$ contient un horizon $\mathrm{B}$ qui répond aux critères de I'horizon podzolique Bf (COMMISSION CANADIENNE DE PÉDOLOGIE, 1978). En effet, la somme de (Fe + Al) extrait par le pyrophosphate dépasse $0,6 \%$ et le rapport $(\mathrm{Fe}+\mathrm{Al}) \mathrm{p}$ / argile est égal à 0,054 . Morphologiquement, la couleur humide répond aux critères de I'horizon $\mathrm{Bf}$, mais la structure polyé- 
TABLEAU ।

Propriétés physiques et chimiques des profils

\begin{tabular}{|c|c|c|c|c|c|c|c|c|c|c|c|c|c|c|}
\hline \multirow[t]{2}{*}{ Horizon } & \multirow{2}{*}{$\begin{array}{l}\text { Profondeur } \\
\text { (cm) }\end{array}$} & \multicolumn{3}{|c|}{ Texture } & \multirow{2}{*}{$\begin{array}{c}\mathrm{CaCO}_{3} \\
(\%)\end{array}$} & \multirow{2}{*}{$\begin{array}{c}\mathrm{pH} \\
\left(\mathrm{CaCl}_{2}\right)\end{array}$} & \multirow{2}{*}{$\begin{array}{c}\text { C orga- } \\
\text { nique } \\
(\%)\end{array}$} & \multicolumn{2}{|c|}{$\mathrm{Fe}$} & \multicolumn{2}{|c|}{$\mathrm{Al}$} & \multicolumn{2}{|l|}{ CEC } & \multirow{2}{*}{$\begin{array}{c}\Sigma \text { bases } \\
\text { échangeables } \\
(\mathrm{meq} / 100 \mathrm{~g})\end{array}$} \\
\hline & & $\begin{array}{c}\mathrm{S} \\
(\%)\end{array}$ & $\begin{array}{c}\mathrm{L} \\
(\%)\end{array}$ & $\begin{array}{c}\text { A } \\
(\%)\end{array}$ & & & & $\begin{array}{c}\mathrm{DCB}^{*} \\
(\%)\end{array}$ & $\begin{array}{l}P Y^{*} \\
(\%)\end{array}$ & $\begin{array}{l}\text { DCB } \\
(\%)\end{array}$ & $\begin{array}{l}\text { PY } \\
(\%)\end{array}$ & $\begin{array}{l}\text { Sol Argile } \\
(\mathrm{meq} / 100\end{array}$ & $\begin{array}{l}\text { Limon } \\
\text { g) }\end{array}$ & \\
\hline
\end{tabular}

Profil Melbourne

Site $n^{\circ} 1$

\begin{tabular}{|c|c|c|c|c|c|c|c|c|c|c|c|c|c|c|c|}
\hline Ap & $0-21$ & 46 & 45 & 9 & - & 5,90 & 2,5 & 1,90 & 0,55 & 0,38 & 0,26 & 13,5 & 30,0 & 4,4 & 6,3 \\
\hline $\mathrm{Bft}$ & $21-43$ & 9 & 72 & 19 & - & 5,48 & 1,1 & 5,45 & 0,71 & 0,48 & 0,32 & 7,3 & 13,7 & 3,7 & 3,6 \\
\hline $\mathrm{BC}_{1}$ & $43-62$ & 6 & 80 & 14 & - & 5,48 & 0,6 & 4,80 & 0,40 & 0,30 & 0,16 & 5,8 & 9,5 & 3,4 & 2,7 \\
\hline $\mathrm{BC}_{2}$ & $62-81$ & 5 & 81 & 14 & - & 6,19 & 0,5 & 5,80 & 0,25 & 0,23 & 0,11 & 5,5 & 11,0 & 3,3 & 5,0 \\
\hline Ck & $81-100$ & 9 & 81 & 10 & 39 & 7,46 & 0,2 & 2,90 & 0,05 & 0,05 & 0,04 & 1,8 & 10,7 & 2,8 & 2,8 \\
\hline Rk & $100+$ & 25 & 72 & 3 & 42 & 7,66 & 0,3 & 1,00 & 0,08 & 0.05 & 0.03 & 1.9 & 8.7 & 3,2 & 2.5 \\
\hline
\end{tabular}

Site $n^{\circ} 2$

\begin{tabular}{|c|c|c|c|c|c|c|c|c|c|c|c|c|c|c|c|}
\hline Ap & $0-20$ & 17 & 57 & 26 & - & 5,71 & 4,3 & 3,15 & 0,48 & 0,30 & 0,18 & 24,3 & 23,3 & 4,6 & 13,6 \\
\hline $\mathrm{Bm}$ & $20-30$ & 6 & 62 & 32 & - & 5,83 & 1,1 & 5,55 & 0,74 & 0,44 & 0,25 & 11,8 & 18,3 & 5,5 & 6,4 \\
\hline BC & $30-58$ & 4 & 68 & 28 & - & 6,46 & 0,5 & 2,75 & 0,22 & 0,20 & 0,07 & 8,4 & 18,7 & 5,1 & 7,8 \\
\hline $\mathrm{Ck}_{i}$ & $58-80$ & 4 & 80 & 16 & 14 & 7,50 & 0,2 & 1,80 & 0,02 & 0,10 & 0,02 & 4,7 & 13,9 & 4,7 & 5,8 \\
\hline $\mathrm{Ck}_{2}$ & $80-110$ & 6 & 77 & 17 & 14 & 7,60 & 0,2 & 1,45 & 0,02 & 0,07 & 0,03 & 3,7 & 10,9 & 7,5 & 4,7 \\
\hline $\mathrm{Rk}$ & $110+$ & 9 & 78 & 13 & 44 & 7,56 & 0,2 & 1,55 & 0,02 & 0,04 & 0,02 & 2,7 & 11,5 & 5,3 & 3 \\
\hline
\end{tabular}

Profil Melbourne-Savoie

$\begin{array}{lrrrrrrrrrrrrrrr}\mathrm{Ap} & 0-14 & 36 & 52 & 12 & - & 6,13 & 9,1 & 2,00 & 0,51 & 0,41 & 0,23 & 43,6 & 31,1 & 6,3 & 23,4 \\ \mathrm{Bg} & 14-24 & 13 & 75 & 12 & \operatorname{tr} & 6,32 & 0,8 & 1,65 & 0,27 & 0,43 & 0,20 & 6,5 & 24,7 & 5,0 & 5,0 \\ \mathrm{Cg}_{1} & 24-34 & 32 & 59 & 9 & \operatorname{tr} & 6,94 & 0,3 & 1,65 & 0,11 & 0,13 & 0,06 & 5,3 & 9,7 & 4,4 & 3,8 \\ \mathrm{Cg}_{2} & 34-50 & 37 & 55 & 8 & \operatorname{tr} & 6,84 & 0,2 & 1,70 & 0,04 & 0,11 & 0,04 & 3,3 & 9,4 & 2,6 & 2,7 \\ \mathrm{Cg}_{3} & 50-56 & 23 & 68 & 9 & \operatorname{tr} & 7,16 & 0,2 & 4,85 & 0,02 & 0,08 & 0,02 & 3,9 & 12,8 & 2,5 & 3,2 \\ \mathrm{Ckg} & 56-80 & 31 & 61 & 8 & 41 & 7,34 & 0,2 & 3,10 & 0,03 & 0,05 & 0,02 & 3,5 & 10,2 & 4,6 & 2,9 \\ \mathrm{Rk} & 80+ & 29 & 65 & 6 & 58 & 7,49 & 0,2 & 1,25 & 0,03 & 0,05 & 0,02 & 2,1 & 8,7 & 3,0 & \end{array}$

Sol organique

\begin{tabular}{lrrrrrrrrrrrrrrr} 
Op & $0-24$ & 60 & 29 & 11 & - & 6,01 & 43,7 & 0,20 & 0,04 & 0,08 & 0,05 & 202,6 & 25,3 & 14,8 & 106,4 \\
$\mathrm{Oh}_{1}$ & $24-32$ & 28 & 57 & 15 & - & 6,12 & 47,9 & 0,53 & 0,24 & 0,06 & 0,08 & 164,6 & 22,0 & 10,7 & 91,5 \\
$\mathrm{Oh}_{2}$ & $32-42$ & 11 & 80 & 9 & - & 6,64 & 43,1 & 0,40 & 0,24 & 0,25 & 0,26 & 166,3 & 35,7 & 14,7 & 94,0 \\
Ckg & $42-52$ & 12 & 76 & 12 & 88 & 7,49 & 3,3 & 0,15 & 0,02 & 0,03 & 0,02 & 6,7 & 8,4 & 5,3 & 2,3 \\
IICcag & $52+$ & 80 & 16 & 4 & 17 & 7,03 & 1,8 & 0,18 & 0,12 & 0,01 & 0,02 & 2,6 & 30,0 & 4,5 & 8,9 \\
\hline
\end{tabular}

* DCB: dithionite-citrate-bicarbonate; PY: pyrophosphate.

drique, bien que peu développée, ne rend pas bien compte de l'incorporation des complexes organométalliques à cet horizon. Le profil correspond à un podzol humo-ferrique orthique. Le profil Melbourne échantillonné au site n'2 est plus riche en argile que le précédent. Alors que la somme de (Fe $+\mathrm{Al}$ ) extraits par le pyrophosphate dans l'horizon $\mathrm{B}$ est proche de $1 \%$, le rapport $(\mathrm{Fe}+\mathrm{Al}) \mathrm{p} /$ argile est égal à 0,03. De plus, la structure de cet horizon est aussi polyédrique. Bien qu'il y ait des revêtements argileux très minces et peu apparents à la surface des peds, I'horizon $\mathrm{B}$ ne répond pas aux critères de l'horizon Bt. Ce profil est classé comme un brunisol mélanique.

L'analyse de ces deux profils confirme donc que la série Melbourne comporte une variété de profils qui peuvent chevaucher plusieurs ordres de sols (ROMPRÉ et al., 1984).

Profil intermédiaire Melbourne-Savoie

Dans des conditions de drainage moins favorables que celles associées aux profils Melbourne, on observe peu de changement dans la texture de ce profil qui contient d'ailleurs encore des traces de carbonates jusque dans l'horizon Bmgj. Les valeurs de $\mathrm{pH}$ dans l'eau sont supérieures à 7 et supérieures à 6 dans $\mathrm{CaCl}_{2} 0,01 \mathrm{M}$. Tout comme dans les profils Melbourne, le $\mathrm{Ca}^{2+}$ est le principal ion présent sur les sites d'échange, et le taux de saturation en bases est élevé. La CEC du limon représente environ $30 \%$ de celle de l'argile. Le carbone organique se trouve principalement dans l'horizon Ap. Les quantités de fer extrait par le dithionite-citrate-bicarbonate sont inférieures à $2 \%$ dans la partie supérieure du profil, mais on observe une zone d'accumulation au contact du matériau riche en calcaire, ce qui est relié à la circulation latérale de l'eau.

Les minéraux argileux sont moins transformés que dans les profils Melbourne. La cristallinité et l'abondance de chlorite diminue quelque peu vers la surface, alors que la formation de minéraux interstratifiés illite-smectite est faible. II n'y a pas d'illuviation d'argile vers l'horizon $\mathrm{B}$ et le profil est classé comme un brunisol mélanique gleyifié. 


\section{Sol organique}

ROMPRÉ et al., (1984) ont mentionné la présence de sols organiques dans le piémont des Appalaches, mais ces sols n'ont pas fait l'objet d'une étude particulière dans leur rapport. Le profil échantillonné pour ce travail présente toutefois de l'intérêt par la succession des couches qu'on y trouve. L'ensemble des couches organiques dépasse $40 \mathrm{~cm}$ d'épaisseur et le contenu en carbone organique y est supérieur à $40 \%$.

En profondeur, le matériau est grossier et contient plus de $80 \%$ de sable. La présence d'un tel matériau dans une région où les dépôts meubles sont particulièrement riches en limon indiquerait peut-être la présence d'un ancien chenal, ce qui est possible quand on considère l'étirement de la dépression dans l'axe SO-NE. En milieu lacustre, en effet, plus de débris ayant une texture identique ou plus fine que celle des matériaux environnants se seraient accumulés dans la dépression. Entre le sable et les couches organiques supérieures, on trouve une couche de $\mathrm{CaCO}_{3}$ presque pur, épaisse de $10 \mathrm{~cm}$ là où l'échantillonnage fut effectué. Ce carbonate provient probablement de la décomposition des roches encaissantes et il s'est accumulé sous forme de marne au fond de la dépression, alors que celle-ci était devenue un bassin fermé. On trouve en effet divers coquillages dans ce matériau (F. Pagé, communication personnelle, 1985). Les carbonates se sont quelque peu infiltrés dans le matériau grossier sous-jacent justifiant l'indice ca de I'horizon IICcag et leur présence se réflète aussi dans les caractéristiques chimiques de la matière organique qui s'est accumulée audessus. Les valeurs de $\mathrm{pH}$ mesurées dans l'eau sont en effet supérieures à 6,5. Ce sol organique est classé comme un humisol terrique.

\section{COMPARAISON AVEC LE PROFIL ESPRIT-SAINT (LA-} FLAMME et al., 1973)

Les profils Melbourne étudiés ici et le profil Esprit-Saint étudié dans le comté de Rimouski présentent des similitudes mais aussi des différences importantes. Le fait calcaire est plus accentué dans le comté de Mégantic à cause de la distribution des carbonates au sein de tous les matériaux parentaux. Dès lors, les carbonates marquent davantage l'évolution des profils et agissent comme un facteur favorisant et freinant en même temps la pédogénèse. La dissolution des carbonates par l'eau chargée de $\mathrm{CO}_{2}$ ou par des acides organiques entraînés par l'eau provoque la comminution des schistes et des ardoises en matériau fin plus réactionnel, mais la présence de quantités abondantes de carbonates ralentit la pédogénèse, puisque les carbonates doivent être éliminés avant que l'altération des minéraux puisse commencer. Un seul horizon répond aux critères de I'horizon podzolique. Dans le sol Esprit-Saint, la distribution moins homogène des carbonates a permis une diminution plus rapide des valeurs de $\mathrm{pH}$ qui, dans le $\mathrm{CaCl}_{2} 0,01 \mathrm{M}$, tombaient en-dessous de 5 dans l'horizon en contact avec le calcaire. Ces valeurs sont donc inférieures de plus d'une unité par rapport à celles mesurées dans les profils Melbourne. Le front de la podzolisation suivait donc de très près l'élimination des carbonates, puisqu'un horizon Bft surmontait immédiatement un horizon BC contenant $48 \%$ de $\mathrm{CaCO}_{3}$.
Les nouveaux critères de classification de l'horizon podzolique (COMMISSION CANADIENNE DE PÉDOLOGIE, 1978) sont sans doute plus exigeants que ceux utilisés en 1973 lors de la classification du profil Esprit-Saint. En appliquant les critères actuels à ce profil, une valeur inférieure à 0,05 du rapport $(\mathrm{Fe}+\mathrm{Al}) \mathrm{p}$ /argile aurait conduit à la classification du profil dans l'ordre luvisolique plutôt que podzolique. Une comparaison plus poussée entre ces profils demanderait de connaître les compositions chimiques totales, surtout les teneurs en fer et aluminium dans les formations Bullstrode et Trinité. Les teneurs en $\mathrm{Fe}$ et $\mathrm{Al}$ déterminent la possibilité qu'ont ces éléments de former des complexes organométalliques, ce qui peut modifier considérablement le rapport $(\mathrm{Fe}+\mathrm{Al}) \mathrm{p} /$ argile dont la limite est fixée à 0,05 (DE KIMPE et al., 1984, DE KIMPE et DEJOU, 1986).

\section{CONCLUSIONS}

1) Dans les sols développés sur matériaux calcaires où le carbonate est uniformément réparti dans les matériaux, il y a évacuation assez rapide des carbonates hors du profil et libération de quantités assez abondantes de particules fines qui participent à la formation d'horizons B texturaux. Au Québec, ce sont sans doute des matériaux parmi les plus susceptibles de donner des horizons Bt.

2) Même pour des sols développés sur tills riches en matériaux calcaires, la décomposition rapide des fragments grossiers et leur absence dans le solum, dans des conditions de drainage bon à modérément bon, peuvent donner l'apparence de sols résiduels. Il est important de vérifier la nature du dépôt meuble dans le contexte géographique régional mais aussi dans des profils profonds non perturbés par la cryoturbation.

3) Sous les conditions climatiques qui règnent au Québec, l'altération des minéraux présents dans les matériaux calcaires, même si elle n'est pas poussée, libère souvent des quantités de $\mathrm{Fe}$ et $\mathrm{Al}$ suffisantes pour atteindre les pourcentages requis par la classification dans l'ordre podzologique. Le facteur limitatif pour justifier cette classification est le taux d'illuviation de l'argile dans le profil qui détermine le rapport $(\mathrm{Fe}+\mathrm{Al}) \mathrm{p}$ / argile.

\section{REMERCIEMENTS}

Les auteurs tiennent à exprimer leur gratitude à $M$. D. Carrier, Service de recherches en sols du MAPAQ, pour l'intérêt qu'il a porté à ce travail et les suggestions faites au cours de la rédaction du manuscrit et à MM. Michel Nolin et William Hendershot pour leurs commentaires très pertinents.

\section{RÉFÉRENCES}

ACTON, C.J., BEKE, G.J., DAY, J.H., MacDOUGALL, J.I. et MARCOUX, R. (1978): Itinéraire d'une excursion dans l'Est du Canada, $11^{\mathrm{e}}$ Congrès de la Société internationale de la science du sol, Edmonton, $188 \mathrm{p}$.

ALIAS, L.J. et HERNANDEZ, J. (1981): The clay mineralogy of high mountain soils on carbonate rocks. II. Inceptisols, Anales de Edafologia y Agrobiologia, vol. 40, p. 11-20. 
ATLAS NATIONAL DU CANADA (1972): Les climats des sols, Données d'Agriculture Canada, Ministère de l'Énergie, des Mines et des Ressources, Ottawa.

BARIL, R. et ROCHEFORT, B. (1957) : Étude pédologique du comté de Lotbinière, Province de Québec, Ministère de l'Agriculture du Canada, Ministère de l'Agriculture du Québec et École Supérieure d'Agriculture, Ottawa, $115 \mathrm{p}$.

BENTLEY, C.F., édit. (1979): Photographs and descriptions of some Canadian soils. Vol. 4, $11^{\text {th }}$ International Congress of Soil Science, Publication B79-1, University of Alberta, Edmonton, 98 p.

CHOINIĖRE, L. (1960): Étude pédologique du comté de Drummond, Bulletin technique n`7, Ministère de l'Agriculture du Québec, Québec, $167 \mathrm{p}$.

COMMISSION CANADIENNE DE PÉDOLOGIE (1978): Le système canadien de classification des sols, Publication n¹646, Ministère de l'Agriculture du Canada, Ottawa, $170 \mathrm{p}$.

COOKE, H.C. (1952): Geology of parts of Richmond and Drummondville map-area, Eastern Townships, Québec, Ministère des Mines du Québec, rapport géologique DP 467.

DE KIMPE, C.R. et DEJOU, J. (1986) : Classification of soils developed on basic parent materials in the Canadian and French systems of soil classification, Canadian Journal of Soil Science (sous presse).

DE KIMPE, C.R., LAVERDIĖRE, M.R., DEJOU, J. et LASALLE, P. (1984): Effects of acidic and basic parent materials on formation of some soils in Quebec (Canada), Geoderma, vol. 33, p. 101118.

ENVIRONNEMENT CANADA (1970): Température et Précipitation, 1941-1970, Service de l'environnement atmosphérique, Ottawa.

GLOBENSKY, Y. (1978): Région de Drummondville, rapport géologique n¹92, Ministère des Richesses naturelles, Québec, 107 p.
LAFLAMME, G., BARIL, R. et DE KIMPE, C.R. (1973): Caractérisation d'un podzol humo-ferrique, luvisolique et lithique à Esprit-Saint, comté de Rimouski, Québec, Canadian Journal of Soil Science, vol. 53, p. 145-154.

McKEAGUE, J.A., édit. (1978): Manuel des méthodes d'échantillonnage et d'analyse des sols, Société canadienne de la science du sol, Ottawa, $250 \mathrm{p}$.

McKEAGUE, J.A., BOURBEAU, G.A. et CANN, D.B. (1967): Properties and genesis of a bisequa soil from Cape Breton Island, Canadian Journal of Soil Science, vol. 47, p. 101-110.

McKEAGUE, J.A., GUERTIN, R.K., PAGÉ, F. et VALENTINE, K.W.G. (1978): Micromorphological evidence of illuvial clay in horizons designated Bt in the field, Canadian Journal of Soil Science, vol. 58, p. 179-186.

McKEAGUE, J.A., MILES, N.M., PETERS, T.W. and HOFFMAN, D.W. (1972): A comparison of luvisolic soils from three regions in Canada, Geoderma, vol. 7, p. 49-69.

RAYMOND, R., LAFLAMME, G. et GODBOUT, G. (1976): Pédologie du comté de Portneuf, Bulletin technique no18, Agriculture Québec, Québec, $164 \mathrm{p}$.

RIVARD, R. et DE KIMPE, C.R. (1980): Propriétés de quelques sols riches en graviers dans la région de Québec, Canadian Journal of Soil Science, vol. 60, p. 263-273.

ROMPRÉ, M., LAFLAMME, G., OUELLET, L., CARRIER, D., DUBÉ, J.C. et PAGÉ, F. (1984) : Étude pédologique du comté d'Arthabaska, Ministère de l'Agriculture, des Pêcheries et de l'Alimentaiton du Québec, Québec, $96 \mathrm{p}$.

ST-ARNAUD, R.J. et SUDOM, M.D. (1981): Mineral distribution and weathering in Chernozemic and Luvisolic soils from Central Saskatchewan, Canadian Journal of Soil Science, vol. 61, p. 79-89. 